\title{
Xeroderma Pigmentosum Group G with Severe Neurological Involvement and Features of Cockayne Syndrome in Infancy
}

\author{
DIMITRIOS I. ZAFEIRIOU, FABRIZIO THOREL, ALEXANDER ANDREOU, WIM J. KLEIJER, \\ ANJA RAAMS, VICTOR H. GARRITSEN, NIKOLAOS GOMBAKIS, \\ NICOLAAS G.J. JASPERS, AND STUART G. CLARKSON \\ First Department of Pediatrics, Aristotle University of Thessaloniki, Thessaloniki, Greece [D.I.Z., N.G.]; \\ Department of Neonatology, "Hippokatio" General Hospital, Thessaloniki, Greece [A.A.]; Departments of \\ Clinical Genetics [W.J.K., V.H.G.] and of Cell Biology and Genetics [A.R., N.G.J.J.], Erasmus University, \\ Rotterdam, The Netherlands; and the Department of Genetics and Microbiology, University Medical
} Centre, Geneva, Switzerland [F.T., S.G.C.]

\begin{abstract}
ABST
We describe a premature, small for gestational age infant girl
with micropthalmia, bilateral congenital cataracts, hearing im-
pairment, progressive somatic and neurodevelopmental arrest,
and infantile spasms. She presented a massive photosensitive
reaction with erythema and blistering after minimal sun expo-
sure, which slowly gave place to small skin cancers. Her skin
fibroblasts were 10 -fold more sensitive than normal to UV
exposure due to a severe deficiency in nucleotide excision repair.
By complementation analysis, the patient XPCS4RO was as-
signed to the very rare xeroderma pigmentosum (XP) group G
(XP-G). One allele of her $X P G$ gene contained a $526 \mathrm{C} \rightarrow \mathrm{T}$
transition that changed Gln-176 to a premature UAG stop codon.
Only a minor fraction of XPG mRNA was encoded by this allele.
The second, more significantly expressed $X P G$ allele contained a
$215 \mathrm{C} \rightarrow$ A transversion. This changed the highly conserved
\end{abstract}
Pro-72 to a histidine, a substitution that would be expected to seriously impair the 3 ' endonuclease function of XPG in nucleotide excision repair. In cases suspected of having XP and/or early-onset Cockayne syndrome, extensive DNA repair studies should be performed to reach a correct diagnosis, thereby allowing reliable genetic counseling and prenatal diagnosis. (Pediatr Res 49: 407-412, 2001)

Abbreviations
CS, Cockayne syndrome
NER, nucleotide excision repair
RT-PCR, reverse-transcription PCR
UDS, unscheduled DNA synthesis
XP, xeroderma pigmentosum
XP-G, xeroderma pigmentosum complementation group G

$\mathrm{XP}$ is a rare autosomal recessive disease characterized by increased frequency of sunlight-induced skin cancers (1). The disorder is caused by defective repair of DNA damage that is induced by UV light or other physical or (bio)chemical agents. Seven genes $(X P A-X P G)$ implicated in XP produce proteins that are involved in this process, which is known as NER. CS is another rare recessive disorder with sun sensitivity, short stature, and progressive neurologic degeneration but no cancer predisposition (1). Five genes are implicated in CS and two of these, the CSA and CSB genes, account for the genetic defect in over $90 \%$ of CS patients. The functions of the CSA and CSB proteins are not yet understood, but cells from these CS

Received June 5, 2000; accepted October 6, 2000.

Correspondence: D.I. Zafeiriou, M.D., Ph.D., Egnatia St. 106, GR-54622 Thessaloniki, Greece.

Supported in part by grant no. 31-52777.97 from the Swiss National Science Foundation. patients are unable to preferentially repair DNA damage in the transcribed strand of active genes, a process known as transcription-coupled repair. In addition, there are a small number of reports on patients with signs of both XP and CS. These cases of the rare XP/CS complex syndrome have been assigned to XP complementation groups $\mathrm{B}, \mathrm{D}$, and $\mathrm{G}(2-6)$. The XPB and $X P D$ genes encode helicases that unwind DNA during both transcription and DNA repair. In contrast, the $X P G$ gene encodes an endonuclease that makes the first of two incisions in the damaged DNA strand during NER, and it has no known function in transcription. A major challenge is to understand how the CS clinical phenotype, and defective transcriptioncoupled repair, can arise through mutations in five very different genes. Here we report a remarkable new XP-G case displaying some, but not all, features of CS. We stress the importance of performing DNA repair studies in cases suspected of having early onset CS with or without clinical signs of XP. 


\section{METHODS}

For all studies performed, informed consent has been obtained and the study has been approved by the applicable institutional review board.

Cell lines. Primary fibroblasts from the patient XPCS4RO were cultured at $37^{\circ} \mathrm{C}$ in the presence of $5 \% \mathrm{CO}_{2}$ in regular Ham F10 (GIBCO, Rockville, MD, U.S.A.) or minimum Eagle's medium (Seromed, Berlin, Germany) supplemented with $5 \%$ FCS, $2 \mathrm{mM}$ l-glutamine, 100 units $/ \mathrm{mL}$ penicillin, and 100 $\mu \mathrm{g} / \mathrm{mL}$ streptomycin. Wild-type lymphoblastoid DS2 cells were grown in suspension at $37^{\circ} \mathrm{C}$ in RPMI 1640 medium supplemented with $15 \%$ FCS, 2 mM l-glutamine, 100 units $/ \mathrm{mL}$ penicillin, and $100 \mu \mathrm{g} / \mathrm{mL}$ streptomycin.

DNA repair assays. DNA repair studies were performed using established procedures described in more detail elsewhere $(4,5)$. Briefly, UDS was measured after UV-exposure $\left(16 \mathrm{~J} / \mathrm{m}^{2} ; 254 \mathrm{~nm}\right)$ of cultured fibroblasts followed by radioactive labeling for $2 \mathrm{~h}$ with ${ }^{3} \mathrm{H}$-thymidine, autoradiography, and counting of grains above 50 non-S-phase cells. Similarly, UDS was assayed in homo- and heterokaryons after fusion with Sendai virus of two XP-cell strains, which had previously been labeled with plastic beads of different sizes.

For measurement of the recovery of DNA synthesis, ${ }^{14} \mathrm{C}$ thymidine-prelabeled cells were exposed to various UV doses and $16 \mathrm{~h}$ later were incubated for $2 \mathrm{~h}$ in ${ }^{3} \mathrm{H}$-thymidine. Relative rates of DNA synthesis were assessed from the ${ }^{3} \mathrm{H} /{ }^{14} \mathrm{C}$ ratios obtained by liquid scintillation spectroscopy. Overall rates of RNA synthesis (transcription) were assayed by autoradiography of cultures pulse-labeled with ${ }^{3} \mathrm{H}$-uridine at $16 \mathrm{~h}$ after UV exposure.

UV sensitivity was measured 4-5 d after UV exposure of sparsely seeded petri dish cultures. Numbers of proliferating cells were assessed by scintillation counting of ${ }^{3} \mathrm{H}$-thymidine incorporated in a 3-h pulse label and were expressed as a percentage of unirradiated cultures. This simplified procedure results in UV survival curves indistinguishable from those obtained by standard colony assays (5).

DNA mutation analyses. Poly(A) + RNA was isolated from XPCS4RO fibroblasts with RNeasy mini kits (Qiagen, Valencia, CA, U.S.A.). Full-length $X P G$ cDNA were produced by RT-PCR following the Expand Reverse Transcriptase and Expand High Fidelity instructions (Roche) using primers 5'TTCTCGGCCGCTCTTAGGACGCAGCCGCC and 5'TTTTATGCATCTTTGCGACAAATTCATTACAAATGGC. $X P G$ cDNA was directly sequenced on a LI-COR DNA sequencer (MWG-Biotech, Ebersberg, Germany) using a set of fluorescently labeled IRD800 primers.

Genomic DNA was isolated from XPCS4RO fibroblasts and wild-type DS2 lymphoblasts and segments containing the mutation sites were amplified using the primer pairs 5'CCCAGATATTAGCATTTGG with 5'-TGAAGGCAGTTTTGATGGC and 5'-CCAGTCTTACCCAAGTTCG with 5'TCATGCTTTACTTCAGGGGG. XPCS4RO genomic DNA was also amplified around known polymorphic sites and the PCR products were directly sequenced as described above.

Human XPG nucleotide and protein sequences are given in EMBL/GenBank accession numbers X69978 and P28715, re- spectively (7). For nucleotide numbering, the A of the initiating ATG codon is designated position 1.

Case history: XPCS4RO. We describe a premature infant girl (Fig. 1), small for gestational age, born at $34 \mathrm{wk}$ gestation with a birth weight of $1420 \mathrm{~g}$, micropthalmia, bilateral congenital cataract, and mild respiratory distress syndrome, hospitalized at the Neonatal Intensive Care Unit for a week. At that time she experienced an episode of erythema with massive desquamation of the skin after minimal exposure to phototherapy. Family history and pregnancy were uneventful. An extended investigation (including brain ultrasound and computed tomography, TORCH titles, metabolic work-up, and chromosomal examination) demonstrated no abnormalities except for absent otoacoustic emissions and abnormal brainstem auditory evoked potential findings, indicative of a severe sensorineural deafness for which hearing aids were prescribed. At the age of 3 mo the patient exhibited a massive photosensitive reaction with erythema and blistering after minimal sun exposure, which slowly gave place to small skin cancers. Although not biopsied, these lesions demonstrated typical appearance of skin cancers according to the dermatologists, showing no signs of improvement over time; on the contrary, new identical lesions seemed to appear with every minimal exposure to the sun at the noncovered parts of the body, especially the face. Her subsequent course was indicative of a neurodegenerative disorder: arrested head circumference (microcephaly; head circumference below the $3^{\text {rd }}$ centile) with somatic and neurologic retardation and development of infantile spasms with burstsuppression pattern in EEG at the age of $8 \mathrm{mo}$; her clinical picture was that of a severe psychomotor regression with generalized hypertonia and loss of eye contact. Both computed tomography (Fig. 2A) and brain magnetic resonance imaging (Fig. 2B) demonstrated brain atrophy, and brainstem auditory-evoked potentials (BAEP) studies showed further deterioration of her hearing impairment. Unfortunately, the little patient died at the age of $11 \mathrm{mo}$ from generalized septicaemia. Necropsy was not performed.

\section{RESULTS}

DNA repair studies. The extreme sensitivity of the patient's skin to sunlight was reflected by that of her cultured skin fibroblasts, which displayed a $10 \times$ hypersensitivity to UV exposure (Fig. $3 A$ ), a residual UDS rate of $4 \%$ to $7 \%$ of normal cells (Fig. 3b, bars 1), as well as a strongly reduced ability to recover from UV-induced inhibition of DNA synthesis (Fig. $3 C$ ) and of overall transcription (data not shown), both measured $16 \mathrm{~h}$ after UV exposure. These abnormalities were due to a severe defect in NER. The observed repair parameters are characteristic for XP patients in groups A, B, and G (8).

To establish which gene underlies the defect, complementation studies were performed by measuring UV-induced UDS in heterokaryons obtained after cell fusion. Fully normal repair levels were restored after fusion with XP-B, XP-D (Fig. 3B) and XP-A cells (not shown), but not with XP-G cells (Fig. $3 B$ ), thereby pointing to a defect in the $X P G$ gene.

Mutation analyses. To determine the nature of the XPG defect(s), poly (A)+ RNA from the patient's fibroblasts was reverse-transcribed and the $3.6 \mathrm{~kb} X P G$ cDNA was amplified by PCR. Direct sequencing of the RT-PCR products revealed a 

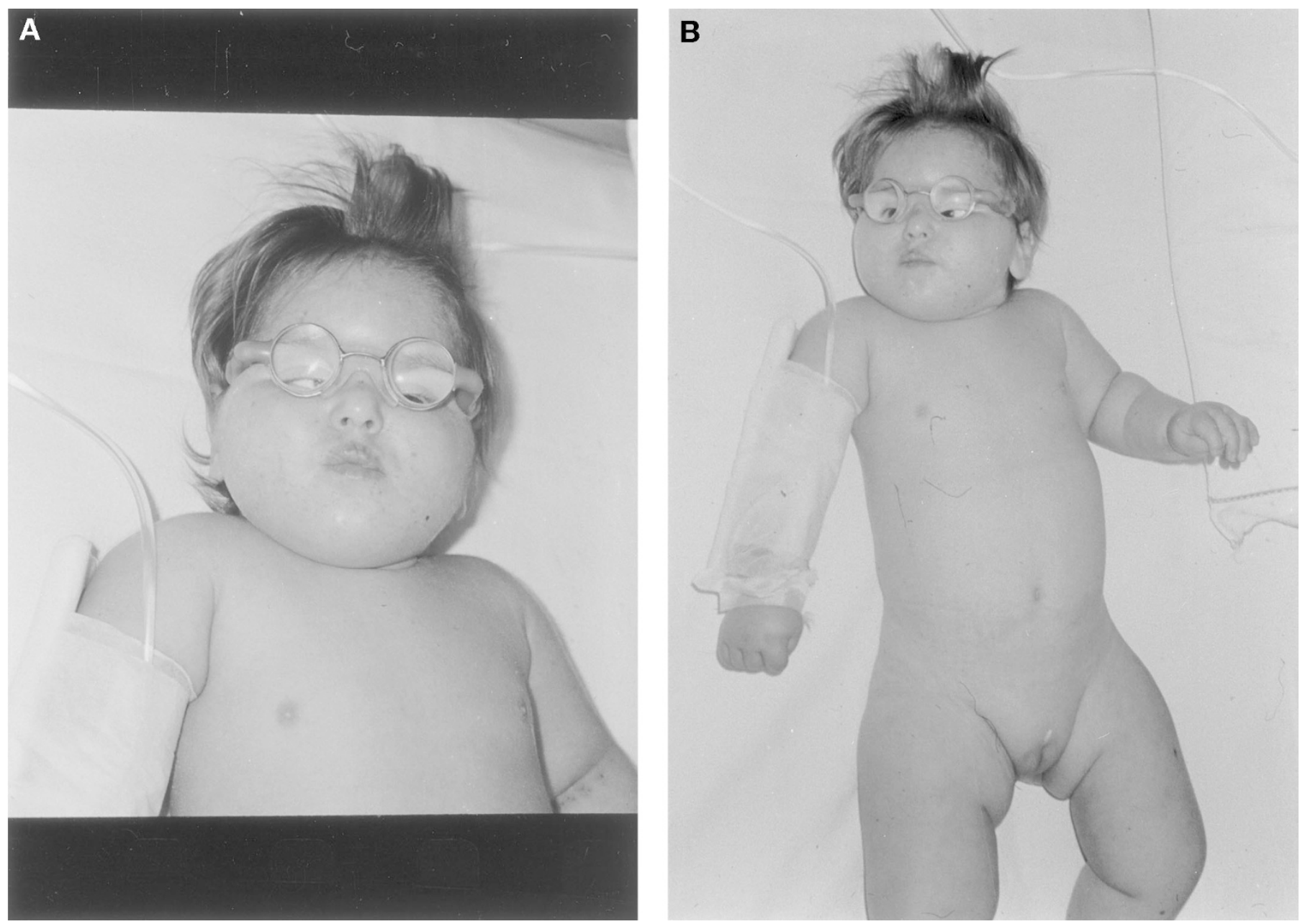

Figure 1. $A$ and $B$ : The index patient at 11 mo of age. Note the somatic retardation, the micropthalmia, and the small skin cancers on the face.

single $215 \mathrm{C} \rightarrow \mathrm{A}$ transversion (arrow in Fig. $4 A$ ). The corresponding region of genomic DNA was amplified by PCR and directly sequenced. Two regions of genomic DNA containing known polymorphic sites (9) were also amplified by PCR and directly sequenced. These analyses revealed that the patient was homozygous for a $\mathrm{G}$ rather than $\mathrm{a} \mathrm{C}$ at position 3310 (data not shown); this changed His-1104 to an alanine. In contrast, she possessed both $\mathrm{C}$ and $\mathrm{T}$ at position 138 (Fig. 4B); this represents a silent polymorphism at His-46. The patient was also heterozygous at position 215 , displaying an equimolar mixture of $\mathrm{C}$ and $\mathrm{A}$, whereas DNA from a normal individual yielded only $215 \mathrm{C}$ (Fig. $4 C$ ).

To examine the possibility that the $215 \mathrm{C}$ allele was weakly expressed in patient XPCS4RO, her RT-PCR products were cloned and 24 independent clones were sequenced. Of the 23 readable sequences, 18 contained the $215 \mathrm{C} \rightarrow$ A mutation but 5 clones had the wild-type $215 \mathrm{C}$. Further analysis of one of these 5 clones revealed the presence of a $\mathrm{C}$ at the polymorphic 138 site, and a 526C $\rightarrow \mathrm{T}$ transition. XPCS4RO genomic DNA generated an equimolar mixture of $\mathrm{C}$ and $\mathrm{T}$ at position 526 (Fig. 4D), thereby confirming the presence of a mutation in the second $X P G$ allele.

We conclude that patient XPCS4RO was a compound heterozygote and that her two $X P G$ alleles can be distinguished in three ways. One allele gives rise to very limited amounts of stable mRNA, it contains a $138 \mathrm{C}$ polymorphism and a $526 \mathrm{C} \rightarrow \mathrm{T}$ transition. This mutation would have changed Gln176 to a premature UAG stop codon, thus resulting in a severely truncated XPG protein. The second allele accounts for most of the XPG mRNA and it contains a 138T polymorphism and a $215 \mathrm{C} \rightarrow \mathrm{A}$ transversion. This mutation gives rise to a $\mathrm{P} 72 \mathrm{H}$ substitution in the conserved N-region of the XPG protein (Fig. 4E).

\section{DISCUSSION}

$\mathrm{XP}$ and CS are very rare autosomal recessive disorders having photosensitivity as one common characteristic, yet only $\mathrm{XP}$ is associated with an extremely high level of skin tumors in sun-exposed areas (1). Neurologic complications represent another of their common features but again they significantly differ, XP patients frequently presenting progressive neurologic degeneration whereas neuronal demyelination is characteristic of CS. The even rarer combination of both disorders, the XP/CS complex, a term first introduced by Robbins (10), affects XP groups B, D, and G, and only a handful of cases are known worldwide. Thus, three out of the five reported XP-B patients present CS (11), only two of the $>60$ XP-D cases are 

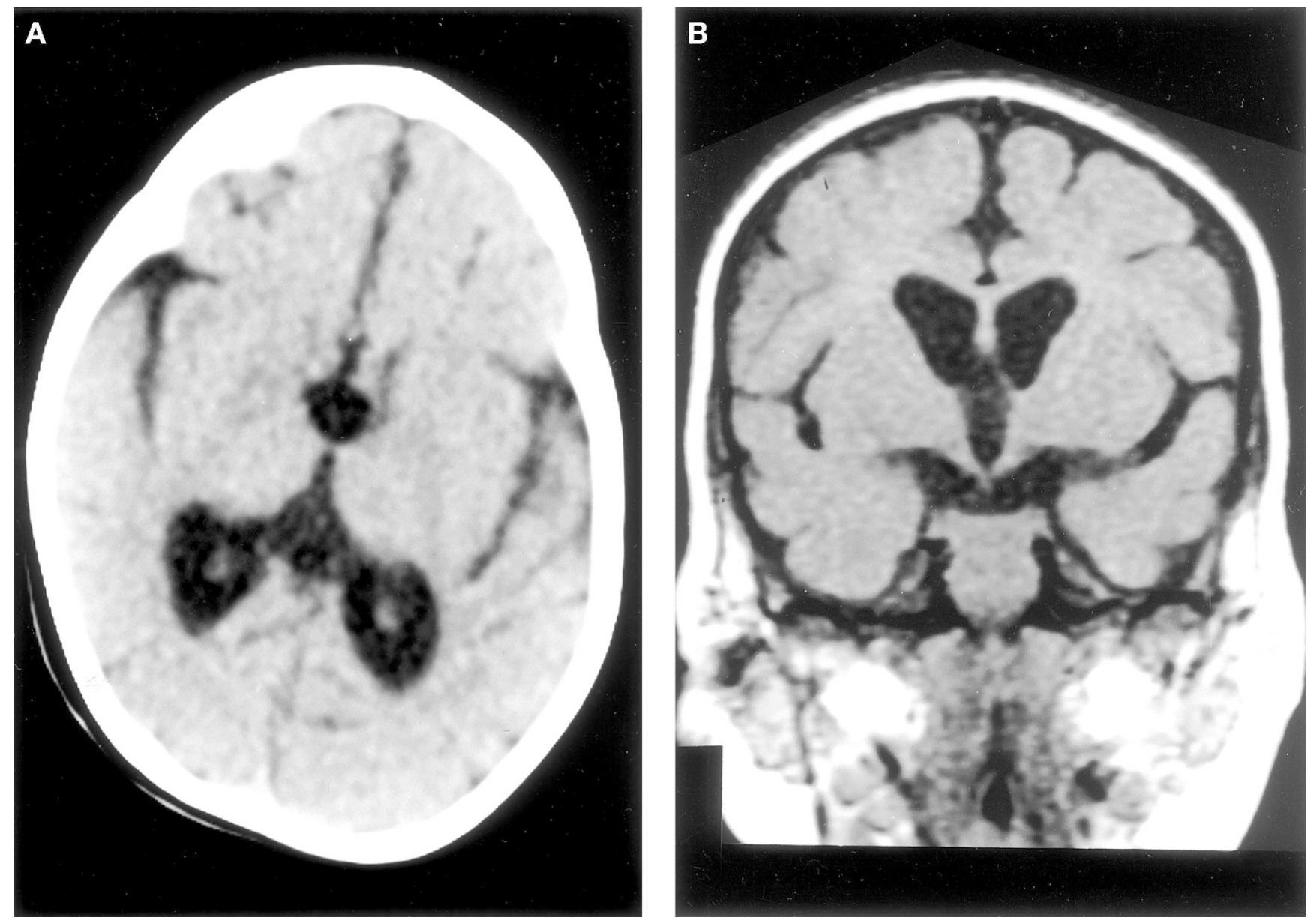

Figure 2. (A) Computed tomography of the brain at $11 \mathrm{mo}$. (B) Magnetic resonance imaging of the brain at 11 mo. Note severe cortical atrophy as well as lateral and 3 rd ventricle enlargement.

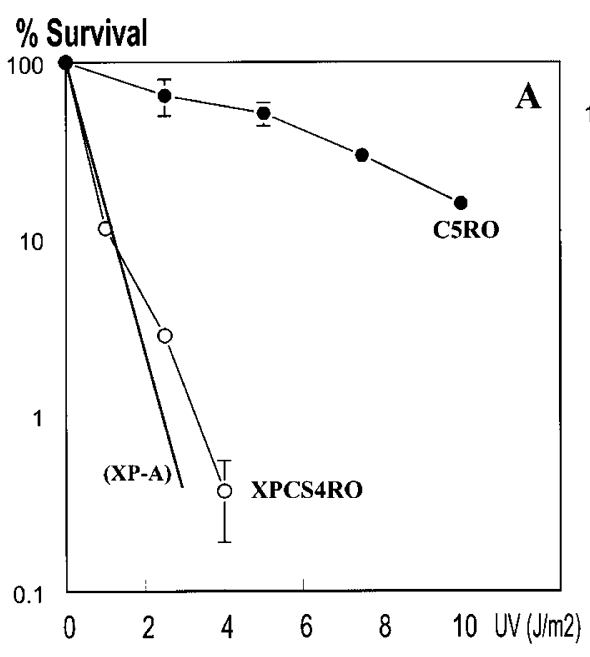

Relative Repair Rate (\%)

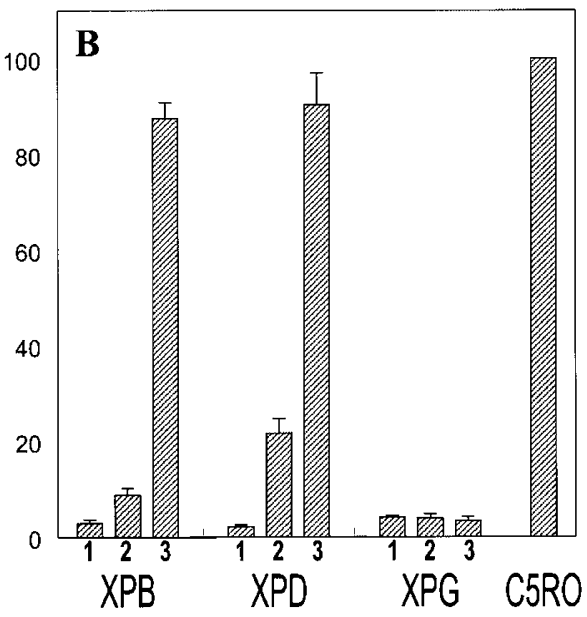

Relative DNA synthesis(\%)

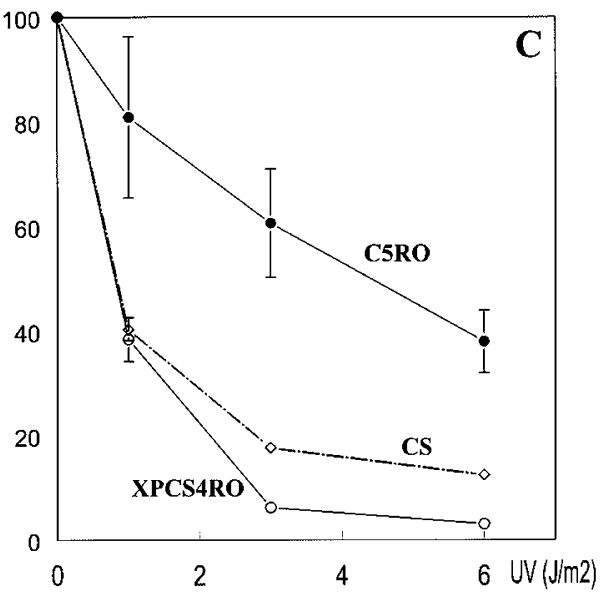

Figure 3. DNA repair characteristics. (A) UV survival curves of fibroblast cultures. SE bars are shown whenever they exceed symbol size. For comparison, a curve of a typical XPA-null fibroblast is added from another experiment. (B) Repair activity measured as UV-induced UDS (\% of normal cells \pm SEM) in binuclear cells. XPCS4RO fibroblasts were fused with either XP-B, XP-D, or XP-G cells and compared with unfused normal cells (C5RO). Bar numbers: $1=$ homodikaryons XPCS4RO; 2 = homodikaryons fusion partner; 3 = heterodikaryons. (C) Recovery of DNA synthesis inhibition $16 \mathrm{~h}$ after exposure to various UV doses.

$\mathrm{XP}-\mathrm{D} / \mathrm{CS}$, and four of the previously reported nine XP-G cases were also CS. The XP-G/CS combination is particularly severe: all four XP-G/CS individuals died prematurely (7 mo to 6.5 y) with few signs of XP (4-6).
The new XP-G patient XPCS4RO reported here is remarkable in many respects. At an exceptionally early age, she presented some hallmark XP features, including severe sun sensitivity with exfoliate blistering, freckling, and keratoses on 
A

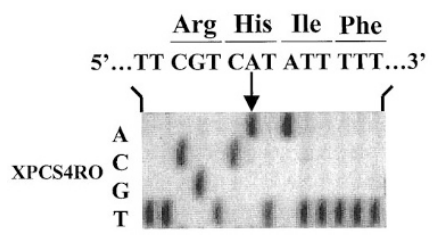

C

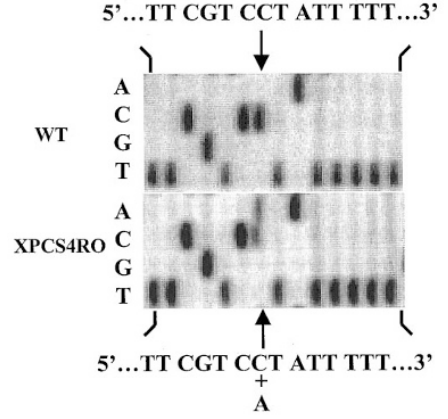

$\mathbf{E}$

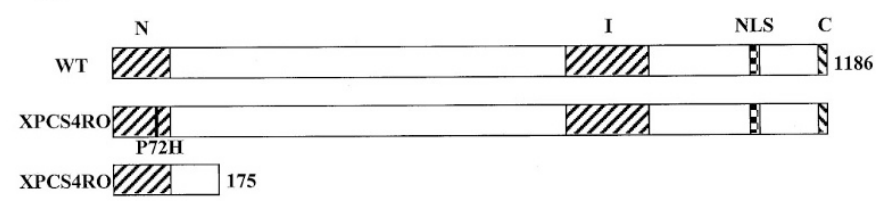

Figure 4. Mutations in patient XPCS4RO. ( $A$ ) Sequence of RT-PCR products showing the $215 \mathrm{C} \rightarrow \mathrm{A}$ transversion resulting in a $\mathrm{P} 72 \mathrm{H}$ amino acid substitution. $(B)$ Sequence of patient genomic DNA showing the mixture of $\mathrm{C}$ and $\mathrm{T}$ at the polymorphic 138 site. (C) Sequence of wild-type (top) and patient (bottom) genomic DNA showing the mixture of $\mathrm{C}$ and $\mathrm{A}$ at position 215 in the patient. (D) Sequence of wild-type (top) and patient (bottom) genomic DNA showing the mixture of $\mathrm{C}$ and $\mathrm{T}$ at position 526 in the patient. The $526 \mathrm{C} \rightarrow \mathrm{T}$ transition in the patient converts Gln-176 to a UAG stop codon. (E) Schematic of wild-type and patient XPG proteins showing the locations of the $\mathrm{P} 72 \mathrm{H}$ and Q176X substitutions. $N$ and $I$, highly conserved N-terminal and internal regions; $N L S$, nuclear localization signal; $C$, basic $\mathrm{C}$-terminus.

sun-exposed parts. Her cultured fibroblasts have very low UDS ability, indicating a severe NER deficiency. She also presented several key CS symptoms, including sensorineural deafness, cataracts, and microcephaly. Additionally, her facial appearance at the age of 11 mo resembled that of a much older child (Fig. 1), which could be an indication of premature aging, a hallmark of CS. Her very early demise at 11 mo might also suggest early onset CS. However, she lacked some other important CS symptoms, such as growth defects, cachexia, a bird-like facies, and brain calcification. Overall, her clinical and cellular phenotypes are consistent with one of two disorders: XP/CS (10), or a particular form of XP, earlier known as De Sanctis-Cacchione Syndrome, which is always associated with neurologic deterioration [reviewed in (12)]. The patient's marked neurodegeneration (atrophy) argues more in favor of De Sanctis-Cacchione Syndrome; however, decreased tendon reflexes usually associated with the latter were not apparent.

The first XP-G patient to be characterized at a molecular level, XP125LO, is a very mildly affected individual currently in her mid-twenties (13). She encodes a truncated XPG protein from her paternal allele and a full-length XPG with an A792V substitution from her maternal allele (9). This substitution occurs in the highly conserved I-region (7) and it severely diminishes the ability of XPG to restore UV resistance to XP-G cells (9). Patient XPCS4RO is also a compound heterozygote with one $X P G$ allele encoding a truncated protein and the other full-length XPG with a single amino acid substitution (Fig. 4). It is curious that mRNA for the truncated form was not detected in the total RT-PCR products (Fig. 4A), whereas it represented 5 of 23 RT-PCR clones. Whatever the explanation, this mRNA accounts for only a minor fraction of the XPCS4RO XPG mRNA and it seems not to be stably maintained. This is consistent with much evidence for the rapid decay of mRNAs containing premature stop codons (14). Attention is therefore focused on the consequences of the mutation in the second $X P G$ allele.

XPCS4RO is unique in being the first XP-G patient to possess an amino acid substitution in the highly conserved $\mathrm{N}$-region (7) and she clearly presented a dramatically different clinical phenotype from that of XP125LO. Her P72H substitution occurs at an invariant position in an important eukaryotic nuclease family (Fig. 5). Human XPG, a founding member of this family, cuts the damaged DNA strand 3 ' to the lesion during NER (15). Recent evidence suggests that the conserved $\mathrm{N}$ - and I-regions (Fig. $4 E$ ) juxtapose to form the XPG endonuclease active site (16). Substitution of Ala for the invariant Asp-77, just five amino acids downstream from the $\mathrm{P} 72 \mathrm{H}$ substitution found in XPCS4RO, abolishes XPG endonuclease function in vivo and in vitro (16). It seems very likely that the $\mathrm{P} 72 \mathrm{H}$ substitution would have an equally dramatic negative effect on XPG endonuclease function, and therefore on the NER capability of patient XPCS4RO.

Although these considerations suggest an adequate explanation for the XP features of this patient, they less convincingly account for her partial CS features. In particular, previous molecular analyses have demonstrated that XP-G/CS individuals are unable to produce full-length XPG protein $(17,18)$. Mildly affected XP-G patients without CS are severely compromised in NER $3^{\prime}$ endonuclease function $(9,16)$. Together, these results have been taken to mean that the CS phenotype is due to the loss of an important second XPG function from these truncated and unstable XPG proteins (17). Additional functions have indeed been found for XPG. In ways not yet understood, it is required for transcription-coupled repair of oxidative DNA damage in vivo (19). In vitro, XPG acts as a co-factor for the DNA glycosylase that removes oxidized pyrimidines in base excision repair (20). Moreover, the latter activity is independent of its endonuclease function. Patient XPCS4RO challenges these correlations because, at first sight, her most significantly expressed allele possesses an "XP-G" rather than an "XP-G/CS" mutation, yet she presented some features typical of CS. This apparent paradox would be resolved if the $\mathrm{P} 72 \mathrm{H}$ substitution destabilised XPG protein. Consistent with this possibility, a vaccinia virus-based overexpression system (16) produces much lower yields of P72H-substituted XPG than wild-type XPG (unpublished results). Unfortunately, the direct experiment of measuring XPG levels in XPCS4RO cell extracts by Western blotting is not yet feasible because available 


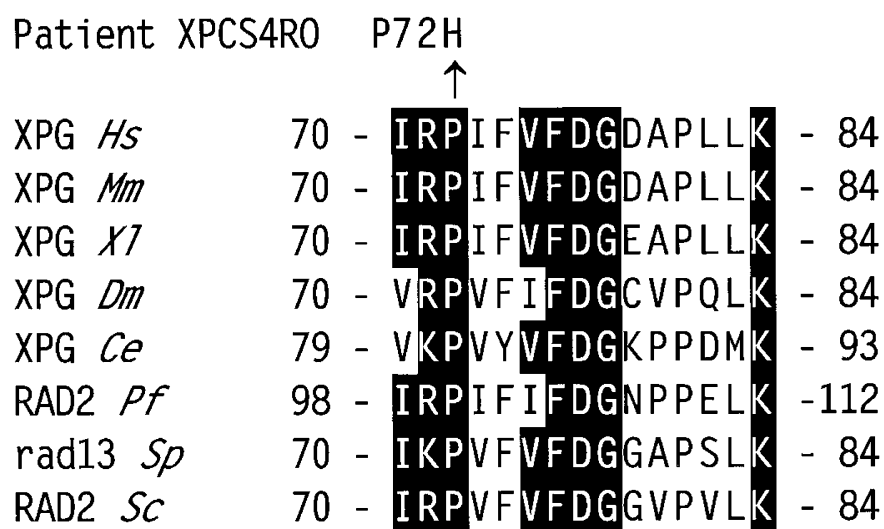

Related enzymes:

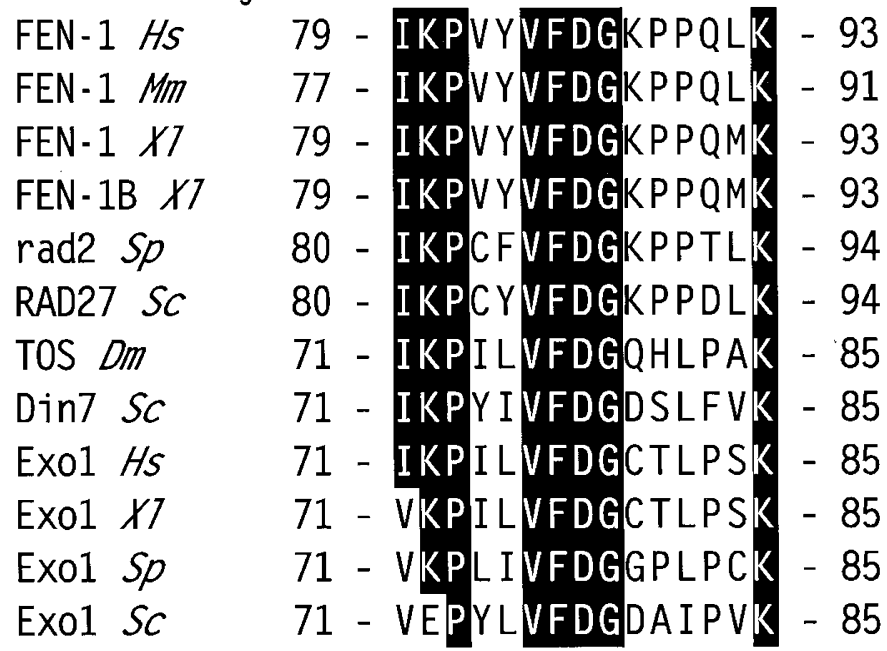

Figure 5. Alignment of the XPG nuclease family within the conserved $\mathrm{N}$-region. The P72H substitution in patient XPCS4RO is aligned with human XPG and its homologues from mouse to yeast. More distant members of this nuclease family are aligned in the lower part of the figure. The sequences (from top to bottom) are from EMBL/GenBank accession numbers P28715, P35689, P14629, AAD47568, AAB96723, E71619, P28706, P07276, P39748, P39749, O57351, P70054, P39750, P26793, Q24558, Q12086, O75466, Q9W6 K2, P53695, and P398875. Abbreviations: Hs, Homo sapiens; Mm, Mus musculus; Xl, Xenopus laevis; Dm, Drosophila melanogaster; Ce, Caenorhabditis elegans; Pf, Plasmodium falciparum; Sp, Schizosaccharomyces pombe; Sc, Saccharomyces cerevisiae.

anti-XPG antibodies are not capable of detecting XPG in extracts of wild-type primary fibroblasts (unpublished results).

The DNA repair studies in the present patient allowed us to counsel the parents on the cause of the disease and on its autosomal recessive nature, which implies a recurrence risk of $25 \%$ in subsequent pregnancies. Both the strongly reduced level of UV-induced DNA repair synthesis and the finding of the two disease-causing mutations allow early prenatal diagnosis. However, because the DNA repair assay requires cultured chorionic villus cells, mutation analysis would be preferred as this can be done directly on DNA isolated from uncultured chorionic villi in the 11th to 12th week of pregnancy. Mutation analysis also allows carrier detection in relatives who have a high chance of being heterozygote (i.e. $67 \%$ for sibs of the patient and $50 \%$ for the parents). Generally, carrier detection in family members is not effective as, in spite of their high risk of being carrier, their risk for affected offspring is quite small as long as their partners are not consanguineous. Carrier detection is, however, effective in populations with a tradition of consanguineous marriage and in small closed communities because of the increased risk that the partners carry the same mutation.

In conclusion, this work highlights the importance of extensive DNA repair studies in cases suspected of having early onset CS and/or XP. Such studies are not only needed to reach a correct diagnosis, thereby allowing reliable genetic counseling and prenatal diagnosis, but they may provide novel insight into the molecular bases of these rare disorders.

Acknowledgments. The authors thank George Katzos, M.D., Ph.D., Persephone Augoustidou-Savvopoulou, M.D., Ph.D., and Manolis Hantjipantelis, M.D., for their help with patient care.

\section{REFERENCES}

1. Cleaver JE, Kraemer KH 1995 Xeroderma pigmentosum and Cockayne syndrome. In: Scriver CR, Beaudet AL, Sly WS, Valle D (eds) The Metabolic and Molecular Bases of Inherited Disease, 7th Ed. McGraw-Hill, New York, pp 4393-4419

2. Jaeken J, Klocker H, Schwaiger H, Bellmann R, Hirsch-Kauffmann M, Schweiger M 1989 Clinical and biochemical studies in three patients with severe early infantile Cockayne syndrome. Hum Genet 83:339-346

3. Scott RJ, Itin P, Kleijer WJ, Kolb K, Arlett C, Muller H 1993 Xeroderma pigmentosum-Cockayne syndrome complex in two patients: absence of skin tumors despite severe deficiency of DNA excision repair. J Am Acad Dermatol 29:883-889

4. Vermeulen W, Jaeken J, Jaspers NG, Bootsma D, Hoeijmakers JH 1993 Xeroderma pigmentosum complementation group $\mathrm{G}$ associated with Cockayne syndrome. Am J Hum Genet 53:185-192

5. Hamel BCJ, Raams A, Schuitema-Dijkstra AR, Simons P, van der Burgt I, Jaspers NGJ, Kleijer WJ 1996 Xeroderma pigmentosum-Cockayne syndrome complex: a further case. J Med Genet 33:607-610

6. Moriwaki S, Stefanini M, Lehmann AR, Hoeijmakers JH, Robbins JH, Rapin I, Botta E, Tanganelli B, Vermeulen W, Broughton BC, Kraemer KH 1996 DNA repair and ultraviolet mutagenesis in cells from a new patient with xeroderma pigmentosum group G and Cockayne syndrome. J Invest Dermatol 107:647-653

7. Scherly D, Nouspikel T, Corlet J, Ucla C, Bairoch A, Clarkson SG 1993 Complementation of the DNA repair defect in xeroderma pigmentosum group $\mathrm{G}$ cells by a human cDNA related to yeast RAD2. Nature 363:182-185

8. Bootsma D, Kraemer KH, Cleaver JE, Hoeijmakers JHJ 1998 Nucleotide excision repair syndromes: xeroderma pigmentosum, Cockayne syndrome, and trichothiodystrophy. In: Vogelstein V, Kinzer K (eds) The Genetic Basis of Human Cancer. McGraw-Hill, New York, pp 245-274

9. Nouspikel T, Clarkson SG 1994 Mutations that disable the DNA repair gene XPG in a xeroderma pigmentosum group patient. Hum Mol Genet 3:963-967

10. Robbins JH 1988 Xeroderma pigmentosum defective DNA repair causes skin cancer and degeneration. JAMA 260:384-388

11. Weeda G, Eveno E, Donker I, Vermeulen W, Chevallier-Lagente O, Taïeb A, Stary A, Hoeijmakers JHJ, Mezzina M, Sarasin A 1997 A mutation in the XPB/ERCC3 DNA repair transcription gene, associated with trichothiodystrophy. Am J Hum Genet 60:320-329

12. Online Mendelian Inheritance in Man, OMIMTM Johns Hopkins University, Baltimore, MD 2000 De Sanctis-Cacchione syndrome. MIM number: 278800. Available at: http://www.ncbi.nlm.nih.gov/omim/

13. Norris PG, Hawk JLM, Avery JA, Gianelli F 1987 Xeroderma pigmentosum complementation group G-report of two cases. Br J Dermatol 116:861-866

14. Hentze MW, Kulozik AE 1999 A perfect message: RNA surveillance and nonsensemediated decay. Cell 96:307-310

15. O'Donovan A, Davies AA, Moggs JG, West SC, Wood RD 1994 XPG endonuclease makes the 3' incision in human DNA nucleotide excision repair. Nature 371:432-435

16. Constantinou A, Gunz D, Evans E, Lalle P, Bates PA, Wood RD, Clarkson SG 1999 Conserved residues of human XPG protein important for nuclease activity and function in nucleotide excision repair. J Biol Chem 274:5637-5648

17. Nouspikel T, Lalle P, Leadon SA, Cooper PK, Clarkson SG 1997 A common mutational pattern in Cockayne syndrome patients from xeroderma pigmentosum group G: implications for a second XPG function. Proc Natl Acad Sci U S A 94:3116-3121

18. Okinaka RT, Perez-Castro AV, Sena A, Laubscher K, Strniste GF, Park MS, Hernandez R, MacInnes MA, Kraemer KH 1997 Heritable genetic alterations in a xeroderma pigmentosum group G/Cockayne syndrome pedigree. Mutat Res 385:107-114

19. Cooper PK, Nouspikel T, Clarkson SG, Leadon SA 1997 Defective transcriptioncoupled repair of oxidative base damage in Cockayne syndrome patients from XP group G. Science 275:990-993

20. Klungland A, Höss M, Gunz D, Constantinou A, Clarkson SG, Doetsch PW, Bolton PH, Wood RD, Lindahl T 1999 Base excision repair of oxidative DNA damage activated by XPG protein. Mol Cell 3:33-42 